\title{
LXXI. On the method in the Trigonometrical Survey for finding the difference of longitude of two stations very little different in latitude
}

\author{
J. Ivory Esq. M.A. F.R.S.
}

To cite this article: J. Ivory Esq. M.A. F.R.S. (1828) LXXI. On the method in the Trigonometrical Survey for finding the difference of longitude of two stations very little different in latitude, Philosophical Magazine Series 2, 4:24, 432-435, DOI: 10.1080/14786442808674883

To link to this article: http://dx.doi.org/10.1080/14786442808674883

曲 Published online: 10 Jul 2009.

Submit your article to this journal $[\pi$

Џll Article views: 2

Q View related articles $₫$ 
LXXI. On the Method in the Trigonometrical Survey for finding the Difference of Longitude of two Stations very little different in Latitude. By J. Ivory, Esq. M.A. F.R.S. \&c.*

$\mathbf{I}^{\mathrm{N}}$ $\mathrm{N}$ this Journal for October last, I endeavoured to prove that the method in the Trigonometrical Survey for finding the difference of longitude of two stations, not much different in latitude, was insufficient, and led to erroneous results. The principle of the method is this, That the latitudes being the same, the difference of longitude is independent of the excentricity,or it is the same on the surface of a sphere and a spheroid of small oblateness; which, in reality, is consistent neither with experience, nor with other methods of investigation of undoubted accuracy. We are told indeed, that the incorrectness of the method, and its want of success in practice, is now allowed on all hands; but the date at which the delusion was dissipated is not mentioned. As my intention was merely to overturn an insufficient method of calculation, not to establish a new rule, I neglected such small quantities as could not be distinguished from the unavoidable errors of observation. Thus the small quantity in the value of $\beta$, which I neglected (p. 243), would be produced by a small variation in the length of the chord $\gamma$, amounting to about 18 feet in the distance between Beachy Head and Dunnose, more than 65 miles. Assuredly a method of calculation which requires such nice accuracy in the data of observation is not a very solid foundation on which to place any conclusion respecting the figure of the earth. There is also an omission in the equations $(x)$ (p. 243), arising from supposing $\mathrm{R}=a$ (p. 242), which, however, will affect the azimuths only a small fraction of a second in the ordinary circumstances of the problem; that is, when one azimuth is greater, and the other less, than $90^{\circ}$. In the extreme case, of which there is no instance that I know of, when the azimuths are both less than $90^{\circ}$, and nearly equal, the last-mentioned omission will affect the accuracy of my formula, because the quantity neglected does not vanish when the two latitudes become equal. There is, however, no doubt that, in the ordinary circumstances of the problem, my method, which takes the excentricity into account, comes nearer the truth than the method in the Survey, which entirely neglects the figure of the earth; and this seemed sufficient to answer my purpose. But it is not easy to put down an authorized error; although I shall now attempt to accomplish this task by new investigations, to which it will be impossible to object.

* Communicated by the Author. 
Mr. Ivory on the Longitudes of the Trigonometrical Survey. 433

Using the same symbols as in this Journal for October, the two following equations, which are rigorously exact, contain the full solution of this problem; viz.

$$
\left.\begin{array}{l}
\Delta=\sqrt{1-e^{2} \sin ^{2} \lambda}, \Delta^{\prime}=\sqrt{1-e^{2} \sin ^{2} \lambda^{\prime}}, Q=\frac{\sin \lambda}{\Delta}-\frac{\sin \lambda^{\prime}}{\Delta^{\prime}} \\
\frac{\sin \omega}{\tan m}+\cos \omega \sin \lambda-\cos \lambda \tan \lambda^{\prime}=\frac{\cos \lambda}{\cos \lambda^{\prime}} \cdot e^{8} \Delta^{\prime} Q \\
\frac{\sin \omega}{\tan m^{\prime}}+\cos \omega \sin \lambda^{\prime}-\cos \lambda^{\prime} \tan \lambda=-\frac{\cos \lambda^{\prime}}{\cos \lambda} \cdot e^{2} \Delta Q
\end{array}\right\}
$$

These equations express the condition that two vertical planes, one at each station, intersect in the chord joining the stations. As the investigation is merely elementary, it may be omitted. The two equations, although very simple, are alone sufficient for the solution of the problem, since the excentricity and the difference of longitude are the only unknown quantities they contain. In order to simplify, I shall put $x=\sin \lambda-\sin \lambda^{\prime}$; and I shall write $\frac{\cos \lambda}{\cos \lambda^{\prime}} \cdot e^{2} x$ and $\frac{\cos \lambda^{\prime}}{\cos \lambda} \cdot e^{2} x$, for the quantities on the right-hand sides. The equations coincide with the surface of a sphere when $e^{q}=0$, and when $x=0$; and as $x$ is always very small in the practical application of the problem, it is with difficulty that the quantities on the right-hand sides enable us to distinguish between the sphere and a spheroid of small oblateness. It is here indeed that the difficulty of the problem lies; and it will easily be conceived that, without nice discrimination, one case is apt to be confounded with the other, as it actually is in the method of the Trigonometrical Survey. In order to solve the equations so as to give full effect to the excentricity of the spheroid, it would be requisite to free them from the almost evanescent factor $x$; but this is what I shall not at present attempt to accomplish. As I write in haste, I shall not inquire how the value of the excentricity is to be deduced, but shall confine my attention to the difference of longitude, supposing that the observations have been made upon a spheroid of a known figure.

The two equations may be brought to a form fit for calculation by the following transformation: viz.

$$
\left.\begin{array}{c}
\tan u=-\frac{1}{\sin \lambda \tan m}, \quad \tan u^{\prime}=\frac{1}{\sin \lambda^{\prime} \tan m^{\prime}}, \\
\frac{\cos (u+\omega)}{\cos u}=\frac{\tan \lambda^{\prime}}{\tan \lambda}\left(1+\frac{e^{2} x}{\sin \lambda^{\prime}}\right), \\
\frac{\cos \left(u^{\prime}-\omega\right)}{\cos u^{\prime}}=\frac{\tan \lambda}{\tan \lambda^{\prime}}\left(1-\frac{e^{2} x}{\sin \lambda}\right) .
\end{array}\right\}
$$

New Series. Vol. 4. No, 24. Dec. 1828. 
434 Mr. Ivory on the Longitudes of the Trigonometrical Survey.

With the data at Beachy Head and Dunnose, and making $\frac{e^{2}}{2}=00324$, I have deduced these values of $\omega$, viz.

$$
\begin{aligned}
& \omega=1^{\circ} 26^{\prime} 58^{\prime \prime} \cdot 3 \\
& \omega=1 \quad 27 \quad 0 \cdot 8
\end{aligned}
$$

These results approach near the true quantity, but they do not agree, which shows that there is some inconsistency in the observed quantities. If we add $0^{\prime \prime} \cdot 6$ to the difference of latitude, the formulas will give results very nearly equal to one another and to the true quantity. Thus, making

$$
\begin{array}{llr}
\lambda_{i}=50^{\circ} & 44^{\prime} & 21^{\prime \prime} \cdot 3 \\
\lambda^{\prime}=50 & 37 & 4 \cdot 7,
\end{array}
$$

I have found $\quad \omega=1 \quad 27 \quad 3 \cdot 8$

$$
\omega=1 \quad 27 \quad 7 \cdot 2 \text {, }
$$

and the mean of these values almost coincides with the exact quantity.

What has now been said is decisive of this question. The equations to which the problem has been reduced, which are rigorously exact, prove that the excentricity has an influence on the difference of longitude, however minute that difference may be, and however difficult to bring it to an exact valuation. The investigation we have employed is drawn from the principles of elementary geometry. We have advanced no vague reasoning about the well-known properties of spheroidical triangles and geodetical lines, in a case where, in fact, there is neither any such triangle nor any such line.

If we combine the two equations (A) so as to exterminate the excentricity, we shall obtain the following equation; viz.

$$
\begin{aligned}
& 0=\sin \omega\left(\frac{\cos \lambda^{\prime}}{\cos \lambda \tan m}+\frac{\cos \lambda}{\cos \lambda^{\prime} \tan m^{\prime}}\right) \\
& +\cos \omega\left(\cos \lambda^{\prime} \tan \lambda+\cos \lambda \tan \lambda^{\prime}\right)-\left(\sin \lambda+\sin \lambda^{\prime}\right) .
\end{aligned}
$$

Here, then, we have an equation which is independent of the excentricity, and which therefore expresses a property common to the sphere and to any spheroid. But if any one should imagine that, now certainly by this exact equation, the difference of longitude may be found independently of the excentricity, he is advised to consider well the principles on which he proceeds before he begins to calculate, lest he should lose his labour.

Dr. Tiarks has treated of this subject in the last Number of this Journal. $\mathrm{He}$ is fortunate enough to take the right side of the question, standing forth as the champion of the method in the Survey. But he has entirely mistaken the nature of the problem and the difficulties that must be overcome in solving 
it. There is no spheroidical triangle; there is no geodetical line. The data consist of the latitudes, nearly equal, and the azimuths, of two distant stations, yet sufficiently near for one to be seen from the other. Now in the case of such distant stations, the observed azimuths are not the same with the azimuths in an imaginary geodetical line passing through the stations. In this, and in other respects, there is great want of precision and geometrical rigour in Dr. Tiarks' argumentation. He has, in fact, brought forward nothing new either in proof, or in elucidation of the problem of which he treats; he has merely added the name of Dr. Tiarks to the other authorities that have upheld the correctness of the method of calculation in the Survey, which it is, nevertheless, just to characterize as the greatest delusion that has ever prevailed in practical mathematics. I can add nothing more on this subject at present; but on another occasion I will give another solution of the same problem, more nearly allied in point of form to the speculations of Dr. Tiarks, which will enable us better to appreciate their merits. When the principles of the problem are fully unfolded, we may then discuss the question, Whether the calculations in the Survey are correct or not?

Nov, 1828.

J. Ivory.

LXXII. Notice of the Geological Features of a Route from Madras to Bellary, in April and May 1822. By Captain W. Cullen, of the East India Company's Artillery service.

[Concluded from p. 363.]

TTHERE are two instances of the occurrence of a rather remarkable variety of quartzose rock in the course of the preceding route, which have not been yet noticed, because it differed very sensibly from any of the others, both in its colour and composition, and appeared likewise to be altogether independent of them. The first occurred on the west side of the pass between Baukrapett and Cuddapah, occupying about one mile of the route, and appearing to constitute the hills on both sides. The lower end of the line of hills, crossed three or four miles before reaching Nundialpett, were composed of rock precisely similar, and were, I imagine, merely a continuation of those forming the pass. I do not recollect any appearances of stratification in either case, although the course of the range was conformable to the general direction. Perhaps this rock rested on clay-slate, and may be considered as merely one of those transitions, formerly alluded to, from sand- 